\title{
Desplazamiento y nuevos arraigos: Pedro Henríquez Ureña y la revista platense Valoraciones
}

\author{
Carolina SANCHOLUZ \\ CONICET-Universidad Nacional de La Plata
}

\begin{abstract}
RESUMEN
Este artículo procura reflexionar sobre el lugar que ocupó Pedro Henríquez Ureña como colaborador de la revista Valoraciones, editada en La Plata entre septiembre de 1923 y mayo de 1928. Esta publicación -llevada a adelante por jóvenes reformistas universitarios-, se constituye en una plataforma desde la cual Henríquez Ureña recomienza y sostiene la práctica de la escritura ensayística apenas radicado en la Argentina, pudiéndose leer en algunas de sus intervenciones líneas fundamentales de su pensamiento crítico, que más tarde se afianzan en sus más reconocidas contribuciones a la historia de la literatura latinoamericana: Seis ensayos en busca de nuestra expresión, la Historia de la cultura en la América hispánica y Las corrientes literarias en la América hispánica. La presencia del intelectual dominicano en las diversas secciones de la revista nos permiten analizar no solo el alcance y efectos de sus intervenciones y operaciones críticas al interior de la publicación, sino que además, el estudio de Valoraciones constituye un medio sumamente valioso para entender el funcionamiento y la proyección de redes intelectuales como lugar de encuentros y también enfrentamientos estéticos e ideológicos, muchas veces entre pares jóvenes y sus mayores.
\end{abstract}

Palabras clave: exilio, desplazamiento, intelectual, red, revista

\section{Displacement and new roots: Pedro Henríquez Ureña and the magazine Valoraciones}

\begin{abstract}
The aim of this article is to reflect on the place that Pedro Henríquez Ureña occupied as a contributor of Valoraciones magazine, edited in La Plata city (Buenos Aires, Argentina) between September 1923 and May 1928. This publication, carried out by young reformist university students, is a platform from which Henríquez Ureña, recently settled in Argentina, starts anew and maintains the practice of writing essays. Some of his interventions show the main lines of his critical thinking, which are later consolidated in his most recognized contributions to the history of Latin American Literature: Seis ensayos en busca de nuestra expresión, la Historia de la cultura en la América hispánica y Las corrientes literarias en la América hispánica.The presence of this Dominican intellectual in the various sections of the magazine has allowed us to analyze the impact of his interventions and critical operations on the magazine. Besides, the study of Valoraciones is a highly valuable tool to understand the functioning and projection of intellectual networks as a meeting place and also as a place of aesthetic and ideological clashes, often between pairs of young people and their elders.
\end{abstract}


Key words: exile, displacement, intellectual, network, magazine

SUMARIO: 1.Introducción: la ruta a la Argentina, el desvío platense. 2.Breves notas sobre Valoraciones. 3.Una firma magistral: Pedro Henríquez Ureña en Valoraciones.

\section{Introducción: la ruta a la Argentina, el desvío platense}

“Ahora cuestión mía y reservada: ¿crees que podré quedarme en la Argentina? ¿Cuánto podré ganar y en qué? Dame el dato lo más seguro y posible, y pronto: contéstame esta carta a más tardar a la semana de recibirla, a fin de que la respuesta llegue a tiempo" (Barcia, 1994:64). Pedro Henríquez Ureña escribe estas líneas urgidas, urgentes-, al joven poeta platense Héctor Ripa Alberdi, en una carta fechada en junio de 1922, desde México. ${ }^{1}$ Se trataba en aquel momento de la segunda estadía mexicana de Henríquez Ureña, entre los años 1921 y1923. Su trayectoria previa, reconocida en el campo intelectual posrevolucionario del país, le había abierto las puertas para participar en el proceso de renovación cultural mexicano bajo la presidencia de Álvaro Obregón (1920-1924) y, en particular, al lado de José Vasconcelos, Ministro de Educación, a cargo de la Secretaría de Educación Pública, responsable de un ambicioso programa educativo que reunió a intelectuales y artistas al servicio del Estado. ${ }^{2}$ Sin embargo, ese contexto favorable que antes había franqueado el ingreso de un intelectual extranjero a múltiples actividades culturales y educativas del país huésped, había cambiado drásticamente, con la ruptura tajante entre Vasconcelos y Henríquez Ureña, hacia fines de $1923 .^{3}$

En septiembre de 1921 se había llevado a cabo en México el Primer Congreso Internacional de Estudiantes que reunió a jóvenes reformistas de América Latina. La delegación argentina, presidida por Héctor Ripa Alberdi, presidente asimismo de

${ }^{1}$ Las cartas de Pedro Henríquez Ureña a Héctor Ripa Alberdi fueron incluidas por Pedro L. Barcia en su estudio Pedro Henríquez Ureña y la Argentina.

${ }^{2}$ Pedro Henríquez Ureña llega a México en 1906 por primera vez y rápidamente se convierte en el mentor del Ateneo de la Juventud, espacio central en el proceso de modernización de la cultura mexicana; se destacó también mediante su laboriosa tarea de mediador intelectual como traductor y antólogo además de docente (en 1913 fue nombrado profesor de Literatura Inglesa en la Escuela Nacional de Altos Estudios creada por Justo Sierra). Arcadio Díaz Quiñones señala al respecto que "Henríquez Ureña sí intervino directamente en la redefinición del canon literario mexicano con antologías y ensayos, y con el comienzo de la ‘nacionalización’ de Juan Ruiz de Alarcón en 1913”. Véase Arcadio Díaz Quiñones, "Pedro Henríquez Ureña (1884-1946): la tradición y el exilio" (2006: 205).

${ }^{3}$ Sobre la etapa decisiva de la vida de PHU en México véase el libro de Alfredo Roggiano, Pedro Henríquez Ureña en México. 
la Federación Universitaria Argentina, estaba compuesta por cuatro jóvenes más, entre ellos, Arnaldo Orfila Reynal, con quien Henríquez Ureña mantuviera luego una profunda amistad. ${ }^{4}$ El intelectual dominicano había sido elegido por sus compatriotas estudiantes como representante de su país en el Congreso. El encuentro entre Pedro Henríquez Ureña y los jóvenes argentinos resultó significativo en varios sentidos, entre ellos, el haberse convertido en un punto de inflexión que modificaría su trayectoria vital e intelectual al definir el próximo -y último- destino de su exilio: la Argentina, país donde se radicó desde 1924 hasta su muerte en 1946, con excepción del breve período 1931-1933. ${ }^{5}$

El encuentro entre Ripa Alberdi y Henríquez Ureña en México inició una fecunda e intensa amistad intelectual entre el joven platense que entonces tenía 24 años y el dominicano, casi quince años mayor, sostenida mediante la relación maestrodiscípulo, como así también a través de afinidades estéticas, éticas e ideológicas: el interés compartido en la cultura humanista sin desatender los problemas sociales, la condena del positivismo a favor del idealismo, el anhelo por la construcción de una cofradía intelectual y espiritual hispanoamericana por encima de las fronteras nacionales y nacionalistas, la necesidad de extender y profundizar la calidad educativa a los más diversos sectores de la sociedad. En octubre de 1922 Pedro Henríquez Ureña acompaña a Vasconcelos en un viaje oficial a la Argentina. A través de sus recientes vínculos con los jóvenes reformistas argentinos con quienes había confraternizado en México en el Congreso Internacional de Estudiantes y por iniciativa de Ripa Alberdi y Orfila Reynal, el decano de la Facultad de Humanidades de la Universidad Nacional de La Plata, Ricardo Levene, lo invita a dictar una conferencia que luego se convertirá en uno de los textos más citados del dominicano: "La

${ }^{4}$ Años después, desde la Dirección de Fondo de Cultura Económica de México, Orfila Reynal llevará adelante el proyecto editorial de la famosa Biblioteca Americana que había planeado Pedro Henríquez Ureña. Además de Ripa Alberdi y Orfila Reynal la Delegación argentina al Congreso Internacional de Estudiantes estaba integrada por Enrique Dreyzen, Pablo Vrillaud y Miguel Bomchill. Tenían como objetivo transmitir al estudiantado latinoamericano el mensaje de defensa y profundización de los postulados de la histórica Reforma Universitaria de 1918, de enorme proyección latinoamericana. Véase el importante ensayo de Juan Carlos Portantiero, Estudiantes y política en América Latina.

${ }^{5}$ En el año 1931 retornó a la República Dominicana para ocupar el puesto de Super Intendente General de Enseñanza, hasta el mes de julio de 1933, bajo el gobierno de Rafael Léonidas Trujillo, gestión de la cual dimitió bruscamente para regresar de inmediato a la Argentina. Arcadio Díaz Quiñones llama la atención acerca de algunos aspectos de la vida de Pedro Henríquez Ureña que han quedado deliberadamente velados, como su reticencia a expresar su rechazo a la dictadura de Trujillo. 
utopía de América” ${ }^{6}$. En ese viaje Henríquez Ureña estrechó sus contactos con escritores argentinos reunidos en torno a la revista Nosotros, con la cual había colaborado de manera esporádica en otras ocasiones ${ }^{7}$. También inició gestiones con Ricardo Rojas, entonces Decano de la Facultad de Filosofía y Letras, con la intención de lograr alguna posición futura en la Universidad de Buenos Aires ${ }^{8}$.

Ahora bien, el contacto más firme -en definitiva el que le permitirá a Henríquez Ureña radicarse en la Argentina-, vino, una vez más, de la mano de los jóvenes reformistas platenses. Primero a través de Ripa Alberdi y, luego de su temprana muerte en 1923, a través de la mediación de Arnaldo Orfila Reynal. Fue por su intermedio que el escritor Rafael Alberto Arrieta se vincula con el intelectual dominicano, interviene activamente ante las autoridades universitarias locales y logra que obtenga tres cursos de Literatura en el Colegio Nacional Rafael Hernández de la Universidad Nacional de La Plata. Este trabajo -para alguno de sus contemporáneos menor o subalterno por tratarse de educación media o secundaria-, constituyó sin embargo su fuente de ingresos más alta y estable entre todas las actividades que llevó a cabo en su período argentino. Como observa atinadamente Arcadio Díaz Quiñones "Henríquez Ureña fue un intelectual sin Estado firme, es decir, no contó

${ }^{6}$ Se pueden rastrear diálogos y confluencias ideológicas entre el discurso inaugural pronunciado por Ripa Alberdi en el Primer Congreso Internacional de Estudiantes, titulado "Por la unión moral de América” (reproducido en el número 2 de la revista Valoraciones, año 1924) y La utopía de América, visibles especialmente en lo que hace a la esperanza de una unión superior de América Latina que, con palabras de Rafael Gutiérrez Girardot, implicara una "conciencia de la gran nación hispanoamericana” (p. 91). Véase de Gutiérrez Girardot "Revisión de la historiografía literaria latinoamericana”, en Hacia una historia de la literatura latinoamericana, coordinado por Ana Pizarro, pp. 79-90; y la discusión que sigue en las pp. 90-100. También la edición preparada por Gutiérrez Girardot para la Biblioteca Ayacucho, Pedro Henríquez Ureña, La utopía de América. El famoso discurso de PHU tuvo una primera edición impresa en La Plata, en un folleto que contenía también otro texto Patria de justicia, en el año 1925, como edición de Estudiantina.

${ }^{7}$ Pedro Henríquez Ureña publica su primera colaboración en Nosotros en el año 1913, en el número 45, donde se reprodujo su conferencia "La obra de José Enrique Rodó" que había pronunciado en el Ateneo de la Juventud de México en 1910. Recién en 1919 vuelve a colaborar con Nosotros, manteniendo la continuidad hasta su radicación en nuestro país.

${ }^{8}$ Henríquez Ureña alude a los contratos que la Universidad de Buenos Aires había hecho con profesores extranjeros, entre ellos Américo Castro, pero que solo se habían firmado con europeos y no con profesores latinoamericanos. Más adelante, cuando Amado Alonso obtiene el cargo de Director del Instituto de Filología Hispánica de la Universidad de Buenos Aires se logra el nombramiento de Henríquez Ureña como Secretario. Por un lado la reforma universitaria abogaba por estrechar los vínculos latinoamericanos y latinoamericanistas, pero por otro las instituciones universitarias no mostraban la misma permeabilidad en la composición de sus cuadros docentes. 
con la protección y los privilegios que caracterizan a la ciudad letrada descrita por Ángel Rama” (Díaz Quiñones, 2006: 168) ${ }^{9}$. Sus desplazamientos, su situación de extranjero, en definitiva, lo que Díaz Quiñones va a caracterizar como su posición de outsider, lo obligaron a nuevos comienzos, en tanto cada nuevo exilio, cada nuevo destino de residencia implicaba un recomenzar.

En las páginas que siguen procuro reflexionar sobre el lugar que ocupó Pedro Henríquez Ureña como uno de los colaboradores más prominentes de la revista Valoraciones, publicada en La Plata entre septiembre de 1923 y mayo de 1928. Por un lado, porque las revistas en general constituyen un canal sumamente valioso para entender el funcionamiento y la proyección de redes intelectuales como lugar de encuentros y también enfrentamientos estéticos e ideológicos, muchas veces entre pares jóvenes y sus mayores. ${ }^{10}$ Por otro, porque Valoraciones se constituye en una plataforma desde la cual Pedro Henríquez Ureña recomienza -desde la propuesta analítica de Díaz Quiñones-, la práctica de la escritura ensayística apenas radicado en la Argentina, pudiéndose leer en algunas de sus intervenciones líneas fundamentales de su pensamiento crítico, que más tarde se afianzan en sus más importantes contribuciones a la historia de la literatura latinoamericana: los Seis ensayos en busca de nuestra expresión (1928), la Historia de la cultura en la América hispánica (1947) y Las corrientes literarias en la América hispánica (original en inglés de 1945, traducción al castellano de 1949).

\section{Breves notas sobre Valoraciones}

Antes de avanzar hacia un análisis puntual de alguna de las intervenciones del escritor en las páginas de Valoraciones es necesario reseñar brevemente el alcance que tuvo la revista, que desde su portada se anunciaba como "Revista bimestral de

${ }^{9}$ Agrega Díaz Quiñones: "Su vida fue un viaje, con sus descubrimientos y riesgos, primero cuatro años en Nueva York, varias estancias en Cuba, luego en México, después cuatro años en Minnesota, en cuya universidad fue profesor, y finalmente veinte años en la Argentina. En Cuba publicó su primer libro, Ensayos críticos, en 1905, y sus libros póstumos, con los cuales alcanzó su merecida fama, se publicaron en México. Nunca se expresó sobre el exilio como un acto heroico, pero llegó a ser la experiencia determinante en su vida, y a ratos con cortes desgarradores. Al elaborar sus linajes literarios tuvo siempre presente la larga tradición del exilio, que lo llevó a una considerable ampliación de su territorio y al enfrentamiento con los nacionalismos. Era también su coraza protectora, un habitus adquirido en la tradición dominicana. Esa otra tradición estaba representada, entre otros, por las figuras caribeñas que más admiró: Hostos y Martí” (2006: 168).

${ }^{10}$ Claudio Maíz y Álvaro Fernández Bravo subrayan "la crucial importancia que tienen las revistas para el estudio de las redes intelectuales.” Véase "Introducción. Los sistemas de religación en la literatura”, en el libro que ambos compilan, Episodios en la formación de redes culturales en América Latina (2009: 37). 
Humanidades, Crítica y Polémica”, a la vez que afirmaba su filiación políticoideológica reformista como "Órgano del grupo de estudiantes Renovación de La Plata”. ${ }^{11}$ La publicación surgió por iniciativa de de Héctor Ripa Alberdi, a mediados de 1923, sin embargo, el poeta declinó su dirección y los miembros del grupo acordaron nombrar a Carlos Américo Amaya en su lugar. Amaya fue su director hasta el número 5 (junio de 1925), cuando decidió asumir un compromiso políticoideológico más marcado y emprender el proyecto de la revista Sagitario, junto a Carlos Sánchez Viamonte y Julio V. González. Desde el número 6 hasta el último número publicado, el 12 (mayo de 1928), la revista fue dirigida por Alejandro Korn, quien había auspiciado la creación de Valoraciones y había sido en buena medida su mecenas, a través del apoyo económico constante a lo largo de los cinco años en los que se mantuvo vigente la publicación. Además de la revista, el Grupo de Estudiantes Renovación sostenía otros dos proyectos culturales, muy afines entre sí: un pequeño sello editorial y una compañía teatral muy activa, tal como se advierte por las noticias y publicidad que Valoraciones promovía desde sus páginas.

"Intenciones", primer editorial firmado por Héctor Ripa Alberdi, reafirma un sentimiento juvenil contestatario. Leemos frases tales como "Nuestra actitud es de rebeldía contra los valores gastados que aún perduran, y de afirmación de nuevos valores."; también "la nueva fantasía y ese nuevo pensamiento, que nos llegan traídos por una amplia y poderosa corriente al humanismo, hemos de recoger en estas páginas, afirmando así, sobre una sólida base idealista, nuestra posición estética e ideológica” (Valoraciones 1, 1923: 3, 4). Sin embargo sus artículos, notas, traducciones y reseñas bibliográficas mantienen un perfil marcadamente académico, en un plano que podemos pensar en términos de "cultura alta". En este sentido llama la atención la convivencia en las páginas de la revista de dos generaciones intelectuales, que se podrían caracterizar en términos de la relación maestrodiscípulo. Por una parte encontramos los nombres de los jóvenes del grupo Renovación -los ya mencionados Carlos Amaya, Ripa Alberdi y Orfila Reynal, Luis Aznar (administrador), Guillermo Korn como secretario de redacción y diagramación, Carlos Sánchez Viamonte-, cuyas firmas aparecen con otras que corresponden a colaboradores por fuera del grupo, pertenecientes a generaciones anteriores y que

${ }^{11}$ Información general sobre Valoraciones se encuentra en el volumen coordinado por Héctor Lafleur, Sergio Provenzano y Fernando Alonso, Las revistas literarias argentinas 1893-1967; en el volumen colectivo Universidad "nueva" y ámbitos culturales platenses (año 1963) hay un artículo que describe la revista y reproduce sus índices. Un estudio de interpretación y análisis de las revistas culturales de la década del veinte, bien documentado, a cargo de Fernando D. Rodríguez, "Inicial, Sagitario y Valoraciones. Una aproximación a las letras y la política de la nueva generación americana”, se encuentra en el volumen colectivo editado por Saúl Sosnowski, La cultura de un siglo. América Latina en sus revistas. 
fueron, en un alto número, profesores universitarios que orientan el marco ideológico y estético general de la revista. Entre ello sobresalen Alejandro Korn, Arturo Marasso, Arturo Costa Álvarez, Francisco Romero y Pedro Henríquez Ureña. Para Fernando D. Rodríguez, autor de varios ensayos sobre las revistas y publicaciones de este período, esta particular confluencia entre profesores y discípulos que singulariza a la revista puede pensarse como un "momento de encuentro socrático con los maestros de la generación anterior” (Rodríguez, 1999: 231). De alguna manera Pedro Henríquez Ureña reafirma entre los jóvenes reformistas platenses el rol de "maestro" que ya había ocupado en México, manifiesto en varios testimonios de quienes estuvieron vinculados al dominicano por diversos motivos. La relación maestro-discípulo que Roberto González Echevarría lee en términos de autoridad, en tanto la figura del maestro es la del poseedor y transmisor del saber cultural, atraviesa las páginas de la revista platense, como si los jóvenes del grupo Renovación todavía necesitaran cobijarse bajo el escudo protector de las firmas consagradas. ${ }^{12}$

Fernando Rodríguez subraya que en los artículos de fondo, casi todos firmados por los "mayores", se desarrollan dos temas fundamentales: los problemas filosóficos revisados bajo las líneas del neoidealismo kantiano y del bergsonismo, y por otra parte, el análisis del estado y evolución de las letras hispanoamericanas:

\begin{abstract}
Ambos tópicos constituyen una permanente requisitoria acerca de la construcción de una 'originalidad' argentina y americana que encuentra sus picos más elevados en la vertiente filosófica en 'Nuevas bases' de Alejandro $\operatorname{Korn}^{13}$ y en el plano literario, en 'Caminos de nuestra historia literaria” de Pedro Henríquez Ureña. (Rodríguez, 1999: 223)
\end{abstract}

Recorriendo el índice de la revista, encontramos, por ejemplo, que el número 4 (julio de 1924), está conformado por una serie de artículos centrales que constituyen un volumen monográfico dedicado a la filosofía kantiana. A su vez, esta línea reflexiva, académica, coexiste con notas de corte estrictamente político, como la que Carlos Américo Amaya dedica al movimiento universitario de Córdoba, a las cuales se suman varias otras -siempre firmadas por los miembros más jóvenes del equipo de redacción-, abiertas hacia el americanismo, que analizan las condiciones

${ }^{12}$ Sobre la relación maestro-discípulo sobresale el trabajo de Roberto González Echevarría, "El extraño caso de la estatua parlante: Ariel y la retórica magisterial del ensayo latinoamericano", incluido en su libro La voz de los maestros.

${ }^{13}$ Las "Nuevas Bases” de A. Korno se publicaron en el número 7 de Valoraciones, de 1925; “Caminos de nuestra historia literaria” se publicó en dos entregas, en Valoraciones 6 y 7 , ambos de 1925. 
presentes y futuras de la Reforma como así también los avatares de la política de América Latina, particularmente de países como México y Perú. Si por un lado se vuelve visible en Valoraciones un espacio cada vez más destacado que asume la defensa y profundización de los postulados reformistas de 1918 y que expresa una orientación socialista y antiimperialista, la revista le da un espacio significativo a obras y autores de la vanguardia literaria y artística en sus páginas. De esta manera se incluyen notas críticas de exposiciones de arte, como, por ejemplo, la que Pedro Henríquez Ureña escribe sobre el pintor Emilio Pettoruti, o la publicación de un texto de Borges como "El tamaño de mi esperanza”, la reseña de Calcomanías de Oliverio Girondo, acompañada de una notable caricatura del escritor, reproducciones de Diego Rivera, etcétera. Tanto Inicial. Revista de la Nueva Generación (1923-1926) de Buenos Aires, como la platense Valoraciones, estrecharon vínculos con publicaciones centrales de la vanguardia local - Martín Fierro y Proa, por ejemplo-, con las cuales intentaron constituir entre 1924 y 1925 un "Frente Único de la Juventud" que finalmente solo quedó en proyecto. ${ }^{14}$

Valoraciones mantuvo en sus doce números una diagramación similar. Se componía de una sección de artículos de contenido filosófico, artístico y doctrinario; una sección bastante famosa "Bibliografía" dedicada especialmente a las reseñas críticas de la producción literaria más reciente de la Argentina, de América Latina y también de Europa, sección que se complementa con otra llamada "Noticias" que alterna reseñas sobre libros recibidos, agradecimientos, la transcripción de cartas (se destacan las que el escritor francés Romain Rolland envía al grupo Renovación a través de la figura de su dirigente Carlos A. Amaya) y es donde aparecen las intervenciones críticas del grupo ante sucesos internacionales como la posguerra europea, la dictadura de Primo de Rivera en España y la persecución sufrida por Miguel de Unamuno, el repudio al discurso de Ayacucho de Leopoldo Lugones, el proceso a Ghandi en la India, donde se expresan también solidaridades políticas con dirigentes latinoamericanos como Raúl Haya de la Torre, deportado de Perú en 1923.

En el año 1928, cuando la revista deja de salir, no son únicamente las dificultades económicas las que determinan su cierre. Como señala Fernando Rodríguez no solo en lo que atañe a las publicaciones del período sino especialmente respecto de los jóvenes actores sociales de entonces:

Una forma de intervención política de los jóvenes intelectuales del '20 está tocando a su fin. Por delante queda para ellos o bien el camino de la profesionaliza-

${ }^{14}$ Sobre esta cuestión remitimos a otro trabajo de Fernando Rodríguez, "La dimensión americana en el debate de las vanguardias estéticas en la Argentina de los '20. Los casos de Inicial y Martín Fierro.” 
ción plena en el campo literario o académico o, en ciertos casos, la incorporación a la lucha política dentro de las estructuras partidarias. (Rodríguez, 1999: 245)

\section{Una firma magistral: Pedro Henríquez Ureña en Valoraciones}

La presencia de Pedro Henríquez Ureña en las páginas de Valoraciones resulta destacada y continua. Por primera vez aparece su firma en una nota central "Poeta y luchador" (Valoraciones 2, enero de 1924), en la cual evoca la malograda figura del joven amigo poeta Héctor Ripa Alberdi, inesperadamente fallecido a los 26 años, el 13 de octubre de 1923. Todo el número constituye un homenaje al poeta y dirigente político estudiantil, cuyo protagonismo e intervención pública en el Congreso Internacional de Estudiantes de México había sido central. La nota que publica la revista reproduce el discurso que pronunció Henríquez Ureña en un acto de homenaje a Ripa Alberdi, en la Universidad de México, palabras que según su propio autor precipitaron la ruptura del vínculo cada vez más frágil con Vasconcelos. ${ }^{15}$ Esta primera colaboración, enviada desde México, da inicio a la articulación concreta de una red intelectual que había empezado con la visita de los jóvenes platenses de la Delegación argentina al Congreso. A partir de esa nota enviada, Pedro Henríquez Ureña no publica en los números 3 y 4 también del año 1924 (son los momentos en los cuales prepara su traslado a nuestro país), reanudando su colaboración, sin interrupciones a partir del número 5 , coincidiendo con su desplazamiento a nuestro país y especialmente con su instalación en la ciudad de La Plata. Participa de todas las secciones de la revista, de manera destacada en los artículos principales donde su firma convive con las de Alejandro Korn, Samuel Ramos, Carlos María Onetti, Arturo Costa Álvarez, Jorge Luis Borges, Ricardo Güiraldes, Francisco Romero, Alfonso Reyes, Romain Rolland. La sentida evocación de Ripa Alberdi en "Poeta y luchador" proyecta la figura del poeta platense como un modelo a seguir por las jóvenes generaciones reformistas: "Héctor Ripa Alberdi, entró por sus méritos de hombre de trabajo y estudio, a la cátedra universitaria, pero no para transigir con la reacción, sino para combatir contra ella” (Valoraciones 2, 1924: 95). Al recordar la participación de la delegación argentina en el Congreso, Henríquez Ureña destaca la red de intelectuales que la Reforma hizo posible a través de

${ }^{15}$ En una carta que PHU le escribe a Alfonso Reyes, en diciembre de 1923, le confiesa lo siguiente: "Con Vasconcelos he acabado de romper sin proponérmelo: hubo una velada, de la Secretaría de Educación Pública, en memoria de Héctor Ripa Alberdi, y hablé yo, aludiendo a la revolución universitaria argentina en 1918, porque en ella colaboró Héctor. Vasconcelos dicen que se sintió aludido y ofendido por las cosas que dije”. Véase Obras completas de PHU, tomo V, p. 322 
representantes como Ripa, aludiendo a una posible unión latinoamericana sustentada en una raíz cultural común:

Cuando en 1922 visitamos la ciudad universitaria de La Plata, encontramos el "ambiente mexicano" creado por ellos: no solo los versos de los poetas mexicanos [...] el entusiasmo por las "ideas mexicanas". Desde hace dos años, México es para aquella juventud símbolo de la pujanza con que la América Latina concibe los ideales de una civilización nueva, original, más amplia y generosa que todas. Tal fue la propaganda cordial que de los ideales latino-americanos hizo Héctor Ripa Alberdi. Ante su tumba declaremos, pues, nuestra decisión de trabajar por la magna patria: la América Española. (Valoraciones 2, 1924: 95-96)

De los artículos publicados por Henríquez Ureña en Valoraciones sobresalen la nota dedicada a la obra pictórica de Emilio Pettoruti ("Sobre la obra pictórica de Emilio Pettoruti” en el número 5, de enero de 1925), que reproduce las palabras pronunciadas en la apertura de la exposición celebrada en la Universidad Nacional de La Plata en noviembre de 1924 e incluye ilustraciones del pintor; el ensayo "Caminos de nuestra historia literaria”, publicado en dos partes (la primera en el número 6, junio de 1925, pp. 246-253; la segunda en el número 7, de septiembre de 1925, pp. 27-32); una nota extensa "Hacia un nuevo teatro" ilustrada con fotografías de puestas en escena y escenografías (número 9, marzo de 1926); un estudio riguroso sobre aspectos técnicos de la prosa y el verso castellanos, publicado en tres partes, llamado "En busca del verso puro" (números 10, agosto de 1926, 11, enero de 1927 y 12, mayo de 1928). ${ }^{16}$

Brevemente propongo detenerme en el ensayo mencionado más arriba, "Caminos de nuestra historia literaria”, porque se advierten en sus dos partes algunos puntos constantes del pensamiento crítico del autor, sobre todo en lo que hace a la posibilidad de trazar una historia canónica de la literatura hispanoamericana, más allá de las tradiciones nacionales pero sin excluirlas, como señala Díaz Quiñones ${ }^{17}$. El texto comienza señalando una falta: los únicos intentos de escribir un ensayo

${ }^{16}$ Son muy importantes las reseñas bibliográficas firmadas por PHU, porque reconstruyen un mapa de lectura con varios nombres y autores que luego incorporará a su "canon” literario latinoamericano. En este sentido resulta ejemplar la reseña que realiza sobre la Antología de la poesía moderna 1900-1925 de Julio Noé (número 9, marzo de 1926, pp. 270-274), recogida luego en Seis ensayos en busca de nuestra expresión con el título de “Poesía contemporánea argentina”, donde se propone trazar “el mapa político de la poesía contemporánea argentina”. En la nota dedicada al libro de Noé rescata el valor del "arte de hacer antologías”, actividad destacada en la producción del propio PHU como antólogo.

17 "Caminos de nuestra historia literaria” se incluye luego en Seis ensayos en busca de nuestra expresión, editado por el sello Babel en Buenos Aires, en 1928. 
abarcador de la literatura de la "América española” se han realizado en otras lenguas como el inglés y el alemán. ${ }^{18}$ Destaca el importante desarrollo de las historias literarias nacionales como un esfuerzo fecundo por comprender las identidades de países como Argentina, Chile, Uruguay, Perú, entre otras naciones de América Latina pero aboga asimismo por emprender un proyecto más amplio: "Todos los que en América sentimos el interés de la historia literaria hemos pensado en escribir la nuestra" (Valoraciones 6, 1925: 247). Desde un nosotros "americano" inclusivo le provee a un posible futuro autor de dicha Historia (que será él mismo) algunas observaciones y consejos para que pueda acometer la obra. En primer lugar define una de las pautas clave a la hora de organizar un canon literario, el imprescindible recorte, la inclusión de nombres representativos que Pedro Henríquez Ureña explícitamente menciona: "La historia literaria de la América española debe escribirse organizándola en torno a unos cuantos nombres centrales: Bello, Sarmiento, Montalvo, Martí, Darío, Rodó...” (Valoraciones 6, 1925: 248). Este criterio se repite como una constante de su pensamiento crítico, tal como escribe en sus Seis ensayos en busca de nuestra expresión: "Con sacrificios y hasta con injusticias sumas es como se constituyen las constelaciones de clásicos en todas las literaturas” (Henríquez Ureña, 1928: 41). Sostiene que el carácter nacional de las literaturas no debe ser una rémora a la hora de pensar en un proyecto más abarcador y que éste debe atender a las diferencias y diversidades que permiten distinguir los hispanoamericano de lo español: "No: el asunto es sencillo. Simplifiquémoslo; nuestra literatura se distingue de España porque no puede menos de distinguirse, y eso lo sabe todo observador" (Valoraciones 6, 1925: 249, cursivas del autor). Por otra parte discute el criterio eurocéntrico por el cual se lee la producción literaria de América Latina bajo el preconcepto o estereotipo de la "exuberancia" y el prejuicio "tropical". Desarma con sutileza la perimida argumentación de las zonas templadas y cálidas, para celebrar la riqueza de la diversidad cultural americana. Para Pedro Henríquez Ureña la originalidad americana está en vías de ser constituida y se vincula estrechamente con el proceso de modernización y de democratización del continente:

Pero las situaciones cambian: las naciones serias van dando forma y estabilidad a la cultura, y en ellas las letras se vuelven actividad normal; mientras tanto, en las 'otras naciones', donde las instituciones de cultura, tanto elemental como superior,

${ }^{18}$ Henríquez Ureña se refiere al estudio de Alfred Coester que se conoció primero en inglés Literary History of Spanish America (Nueva York: The Mac Millan Co., 1916) y que circuló a partir de 1929 en español, bajo el nombre de Historia Literaria de la America Española, traducción por Rómulo Tovar, Madrid, Casa Editorial Hernando, 1929, 564 p. Asimismo menciona el trabajo del alemán Max Leopold Wagner, Die Spanisch Amerikanische Literatur in ihren Hauptstrómungen, Leipzig-Berlín: B. G. Teubner,1924. 
son víctimas de los vaivenes políticos y del desorden económico, la literatura ha empezado a flaquear. (Valoraciones 6, 1925: 253, cursivas del autor)

En la segunda entrega del ensayo, como destaca Fernando Rodríguez, se retoma un tópico que atraviesa las publicaciones de la época, propio del clima de ideas de los años veinte, el eclipse o decadencia europea:

Yo no sé si empezaremos a 'ser nosotros mismos' mañana a la aurora o al mediodía; no creo que la tarea histórica de Europa haya concluido; pero sí sé que para nosotros Europa está en eclipse, pierde el papel dogmático que ejerció durante cien años. No es que tengamos brújula propia; es que hemos perdido la ajena ( $\mathrm{Va}$ loraciones 7, 1925: 27).

Frente a esta situación postula un retorno a los orígenes para "aprender a pensar las cosas desde su raíz” (Valoraciones 7, 1925: 28). En ese camino Henríquez Ureña plantea no renunciar al derecho de nuestra cultura a "movernos con libertad dentro de la tradición española, y, cuando podamos, a superarla. Todavía más: tenemos derecho a todos los beneficios de la cultura occidental" (Valoraciones 7, 1925:29). De ahí que defienda asimismo el derecho a la imitación (muy visible en la selección de alguno de sus autores canónicos, Darío, Rodó), como una estrategia de apertura universal de las literaturas nacionales de América Latina: “Cualquier literatura se nutre de influjos extranjeros, de imitaciones y hasta de robos [...]. Pero el caso es grave cuando la transformación no se cumple, cuando la imitación queda en imitación” (Valoraciones 7, 1925: 29). En la última parte del ensayo, significativamente subtitulada "Los tesoros del indio", Henríquez Ureña incorpora la otra herencia del fuerte del espíritu americano: el arte indígena. Para el ensayista este es el "camino" de nuestra historia literaria: nutrirse en las dos fuentes, la española y la indígena, "hasta que el espíritu se revele en nuestras creaciones acrisolado" (Valoraciones 7, 1925: 32). ${ }^{19}$ Esta misma idea reaparece con fuerza en Las corrientes literarias de la América hispánica cuando destaca la convivencia con el "indio", y la creación de "una sociedad nueva” caracterizada por la "fusión de culturas" europeas y precolombinas, como así también en el capítulo inicial de la Historia de la cultura en la América hispánica (publicación póstuma de 1947), titulado "Las culturas indígenas”.

${ }^{19}$ Cabe subraya que no pasamos por alto aquellas ausencias significativas en las construcción de un relato cultural latinoamericano integrador por parte del dominicano, como el lugar y peso de las herencias africanas. En este sentido resultan iluminadores varios pasajes del ensayo de Díaz Quiñones, cuando señala que Henríquez Ureña construye sus beginnings hispánicos a partir del contrapunto con lo afroamericano. Véanse los apartados VI "Las eses del negro culto”, pp. 231-243. 
"Caminos de nuestra historia literaria" cifra de manera condensada algunos aspectos centrales del pensamiento crítico y analítico de Pedro Henríquez Ureña, por ejemplo, la "utopía de América" como una gran nación hispanoamericana, sostenida en una concepción integradora de la cultura de los pueblos latinoamericanos. En su teoría y en su práctica, la cultura hispanoamericana abarcaba la historia literaria y las instituciones. Pero incluía también la historia de las ideas, la historia social, la pintura, la música, y los textos escritos en el marco "nacional”. Para Díaz Quiñones allí es posible advertir una concepción "fuerte" de la cultura como sujeto de la historia y como objeto de estudio, lo que le permitió construir uno de los "grandes relatos” que hacen posible una identidad integradora de América Latina:

La perspectiva histórico-cultural minuciosamente articulada en sus ensayos y antologías activó un circuito de textos y autores que siguen aún en el centro del debate. En las grandes “sumas” publicadas después de su muerte -la Historia de la cultura en la América hispánica (1947) y Las corrientes literarias en la América hispánica $(1945,1949)$ la historia de la "cultura” era signo de pertenencia a una comunidad. (Díaz Quiñones, 2006: 172-173)

Desde las páginas de Valoraciones es posible avizorar la temprana emergencia del sentido de una comunidad cultural latinoamericana amplia en el pensamiento crítico de Pedro Henríquez Ureña, tal como lo destaca Rafael Gutiérrez Girardot cuando afirma en el intelectual dominicano la conciencia de la gran nación hispanoamericana. A través de diálogo fecundo con los jóvenes reformistas universitarios de La Plata, con quienes conformó una cofradía singular, Henríquez Ureña proyectó su figura de "maestro" como una forma posible del arraigo en aquello a lo cual anhelaba contribuir con firmeza: la construcción de una patria cultural america$n a^{20}$

\section{BIBLIOGRAFÍA}

ARRIETA, Rafael Alberto.

1956 "Pedro Henríquez Ureña, profesor en la Argentina”, en Revista Iberoamericana vol. XXI, $\mathrm{n}^{\circ}$ 41-42, enero-diciembre de 1956, pp. 8997.

${ }^{20}$ Colaboración y agradecimiento: los trabajos de archivo bibliográfico sobre la revista Valoraciones fueron llevados a cabo por Alejandra Aracri (Estudiante avanzada de las Carreras de Letras y Bibliotecología en la Facultad de Humanidades y Ciencias de la Educación de la Universidad Nacional de La Plata). 
BARCIA, Pedro Luis.

1994 Pedro Henríquez Ureña y la Argentina. Santo Domingo: Universidad Nacional Pedro Henríquez Ureña.

DÍAZ QUIÑONES, Arcadio

2006 "Pedro Henríquez Ureña (1884-1946): la tradición y el exilio”, en Sobre los principios. Los intelectuales caribeños y la tradición. Quilmes: Universidad Nacional de Quilmes, pp. 167-254.

GÓNZALEZ ECHEVARRÍA, Roberto.

2001 La voz de los maestros. Escritura y autoridad en la literatura latinoamericana moderna. Madrid: Verbum.

HENRÍQUEZ UREÑA DE HLITO, Sonia.

1993 Pedro Henríquez Ureña: apuntes para una biografía. México: Siglo XXI.

HENRÍQUEZ UREÑA, Pedro.

1928 Seis ensayos en busca de nuestra expresión. Babel: Buenos Aires.

1947 Historia de la cultura en la América hispánica. México: Fondo de Cultura Económica.

1949 Las corrientes literarias en la América hispánica. Trad. de Joaquín Diez Canedo. México: Fondo de Cultura Económica.

1978 La utopía de América. Ángel Rama y Rafael Gutiérrez Girardot (comps.), pról. de Rafael Gutiérrez Girardot. Caracas: Ayacucho.

1981 Epistolario íntimo (1906-1946). Epistolario entre PHU y Alfonso Reyes, 3 tomos. Pról. de Juan Jacobo de Lara, Santo Domingo: Universidad Nacional Pedro Henríquez Ureña.

1989 Memoria. Diarios. Edición y notas de Enrique Zuleta Álvarez, Buenos Aires: Academia Argentina de las Letras.

1998 Ensayos. Edición crítica de José Luis Abellán y Ana María Barrenechea. Madrid/París/México: Colección Archivos.

MAÍZ, CLAUDIO y ÁLVARO FERNÁNDEZ BRAVO (eds.).

2009 Episodios en la formación de redes culturales en América Latina. Buenos Aires: Prometeo.

PORTANTIERO, Juan Carlos.

1978 Estudiantes y política en América Latina. México: Siglo XXI.

RODRÍGUEZ, Fernando D.

1999 "Inicial, Sagitario y Valoraciones. Una aproximación a las letras y la política de la nueva generación americana”, en Sosnowski, Saúl (ed.). La cultura de un siglo. América Latina en sus revistas. Buenos Aires: Alianza, 1999, PP. 217-247.

1994 "La dimensión americana en el debate de las vanguardias estéticas en la Argentina de los '20. Los casos de Inicial y Martín Fierro.”, Boletín de Historia, FEPAI, 1994, pp. 3-21. 
ROGGIANO, Alfredo.

1989 Pedro Henríquez Ureña en México. México: UNAM.

SARLO, Beatriz.

1985 “Pedro Henríquez Ureña: lectura de una problemática”, Revista de Filología, XX, Buenos Aires, pp. 9-20.

Valoraciones. Revista bimestral de Humanidades, Crítica y Polémica, 1923-1928

[1-12]. La Plata, Editada por el Grupo de Estudiantes "Renovación”.

ZULETA ÁLVAREZ, Enrique

1997 Pedro Henríquez Ureña y su tiempo. Buenos Aires: Catálogos. 